\title{
ISOBARIC ANALOG RESONANCE PROPERTIES IN A UNIFIED MODEL CALCULATION
}

\author{
G. VANDEN BERGHE \\ Seminarie voor Wiskundige Natuurkunde, \\ Rijksuniversiteit-Gent, Belgium* \\ K. HEYDE ${ }^{* *}$ \\ Fysisch Laboratorium, Rijksuniversiteit-Utrecht, The Netherlands \\ M. WAROQUIER *** \\ Instituut voor Nukleaire Wetenschappen, \\ Rijksuniversiteit-Gent, Belgium \\ Received 22 January 1972
}

\begin{abstract}
Partial-widths for proton inelastic scattering in the reaction ${ }^{140} \mathrm{Ce}\left(p, p^{\prime}\right)$ to the first excited $2^{+}$state are calculated in the framework of a unified model calculation. These values are compared with recent experimental data. A detailed information about the structure of the wavefunctions for levels in ${ }^{141} \mathrm{Ce}$ is given.
\end{abstract}

Great interest has been shown in the study of the properties of the $N=83$ nuclei. The neutron single-particle states have been investigated with (d,p) reactions on the $N=82$ nuclei as target nuclei. $A{ }^{143} \mathrm{Nd}\left(\mathrm{d}, \mathrm{d}^{\prime}\right)$ reaction work gives us information about the collective character of some states. The knowledge available on the electromagnetic decay of the levels in these nuclei essentially comes from $(n, \gamma)$ work on $138 \mathrm{Ba}$, ${ }^{140} \mathrm{Ce}$ and ${ }^{144} \mathrm{Sm}$. A detailed theoretical description of the level schemes, wavefunctions and electromagnetic properties in the framework of a unified model calculation has already been given elsewhere by the authors [1]. (We refer to that article for all references concerning the most important experimental information.)

By studying the isobaric analog states of the levels in the $N=83$ nuclei as resonances by means of proton elastic scattering on the eveneven $N=82$ target nuclei, one can get spectroscopic information, which supplements that obtained from the $(d, p)$ reactions. In this way it was possible to check only the single-particle

* Correspondence address: I.N.W. - Rijksuniversiteit-Gent, Proeftuinstraat 86, B-9000 Gent, Belgium.

** One leave of absence from Instituut voor Nukleaire Wetenschappen, Rijksuniversiteit-Gent, Belgium .

*** Aspirant of the National Fonds voor Wetenschappelijk Onderzoek. component of the wavefunctions to experimental spectroscopic factors. Although the total wavefunctions could be tested by calculating some electromagnetic properties, which could be compared with experimental data, no detailed information was experimentally known about the important collective components of the wavefunctions.

Recently one has determined through inelastic scattering of polarized protons from $140 \mathrm{Ce}$ to the $2^{+}\left(E_{\mathrm{X}}=1.596 \mathrm{MeV}\right)$ state near isobaric analog resonances, the so-called parentage coefficients for six $p$ and $f$ states in ${ }^{14} 1 \mathrm{Ce}[2,3]$. These coefficients are completely identical with the expansion coefficients $C_{\alpha}(l j, N R ; I)$, by which the eigenfunctions at an energy $E^{(\alpha)}$ of a state in ${ }^{141} \mathrm{Ce}$ have been described [1], i.e. $\ddagger$ $\left|E^{(\alpha)} ; I M\right\rangle=\sum_{j N R} C_{\alpha}(l j, N R ; I)|l j, N R ; I M\rangle$.

For further simplicity we denote the basis, in which the wavefunctions for the odd-neutron nucleus is expanded as follows

$$
|l j, N R ; I M\rangle=[n(l j) \otimes C(N R)]^{I M}
$$

where the neutron single-particle state $n(l j)$ is coupled with the $N$-phonon state of the core $C(N R)$ to total angular momentum $I$ and projec-

$\ddagger$ For the meaning of the symbols we refer to ref. [1]. 
tion $M$ along the $z$-axis. To be more specific, we can write

$$
\begin{aligned}
& \left|E^{(\alpha)} ; I M\right\rangle \equiv|n C ; I M\rangle= \\
& \left.\quad C_{\alpha}(l j .00 ; j){ }^{r} n(l j) \otimes C(00)\right]^{j m} \\
& \quad+\sum_{l j} C_{\alpha}(l j, 12 ; I)[n(l j) \otimes C(12)]^{I M} \\
& \quad+\sum_{l j i} C_{\alpha}\left(l j, C_{i}\left(^{*}\right) ; I\right)\left[n(l j) \otimes C_{i}\left({ }^{*}\right)\right]^{I M}
\end{aligned}
$$

where $C_{i}\left(^{*}\right)$ is written down for all other excited states of the $N=82$ core, with exception of the groundstate and the first excited one-quadrupolephonon state. These configurations now represent the parent of the states obtained by inelastic proton scattering experiments on the $N=82$ even-even nuclei. The total isospin and its third component $T_{3}$ have the value $T=\frac{1}{2}(N-Z)$ for the target $N=82$ nucleus in its groundstate. An isobaric analog state $|n C ; I M\rangle_{A}$ is generated by the application of the isospin lowering operator $T_{-}$to $|n C ; I M\rangle$. which changes the $T_{<}$states $\left(T_{3}=T+1 / 2\right)$ of ${ }^{141} \mathrm{Ce}$ into the $T_{>}$states $\left(T_{3}=\right.$ $T-1 / 2)$ of ${ }^{141} \operatorname{Pr}$ [4].

$$
\begin{aligned}
& |n C ; I M\rangle_{A}=\frac{1}{\sqrt{2 T+1}}\left\{\sqrt{S_{l}^{(\alpha)}}[p(l j) \otimes C(00)]^{j m}\right. \\
& \quad+\sum_{l j} C_{\alpha}(l j, 12 ; I)[p(l j) \otimes C(12)]^{I M} \\
& \quad+\sum_{l j i} C_{\alpha}\left(l j, C_{i}\left(^{*}\right) ; I\right)\left[p(l j) \otimes C_{i}\left(^{*}\right)\right]^{I M} \\
& \quad+\sqrt{2 T}\left[\sqrt{S_{l}^{(\alpha)}}[n(l j) \otimes A(00)]^{j m}\right. \\
& \quad+\sum_{l j} C_{\alpha}(l j, 12 ; I)[n(l j) \otimes A(12)]^{I M} \\
& \quad+\sum_{l j i} C_{\alpha}\left(l j, C_{i}{ }^{\left.\left.(*) ; I)\left[n(l j) \otimes A_{i}\left(^{*}\right)\right]^{I M}\right]\right\}} .\right.
\end{aligned}
$$

Here $p(l j)$ denotes the wavefunction of a singleparticle proton state, which is the isobaric analog state of $n(l j) ; A(N R)$ are the isobaric analog states of the $C(N R)$ core states. For simplicity we have denoted $C_{\alpha}(l j, 00 ; j)$ by the square root of the spectroscopic factor $\sqrt{S(\alpha)}[1]$. The first term describes the elastic proton channel; the other terms are connected with the inelastic proton channels, where in the second and in the third term the core in an excited collective state, while the other terms are connected with the situation where we have a neutron hole in the magic neutron core. These states, called generalized neutron particle-hole (GNPH) states are experimentally studied for ${ }^{140} \mathrm{Ce}$ by the Heidelberg group [5]. However these GNPH states are found in ${ }^{140} \mathrm{Ce}$ above $3.12 \mathrm{MeV}$, so that by studying the structure of the low-lying states of ${ }^{141} \mathrm{Ce}$ only the first two terms will be of importance.

The strength of the several exit channels of the isobaric analog resonances depends on the expansion coefficients $C_{\alpha}(l j . N R ; I)$. The spectroscopic factor $S_{l}^{(\alpha)}$ can be determined from elastic proton scattering or from $(d, p)$ reaction experi-

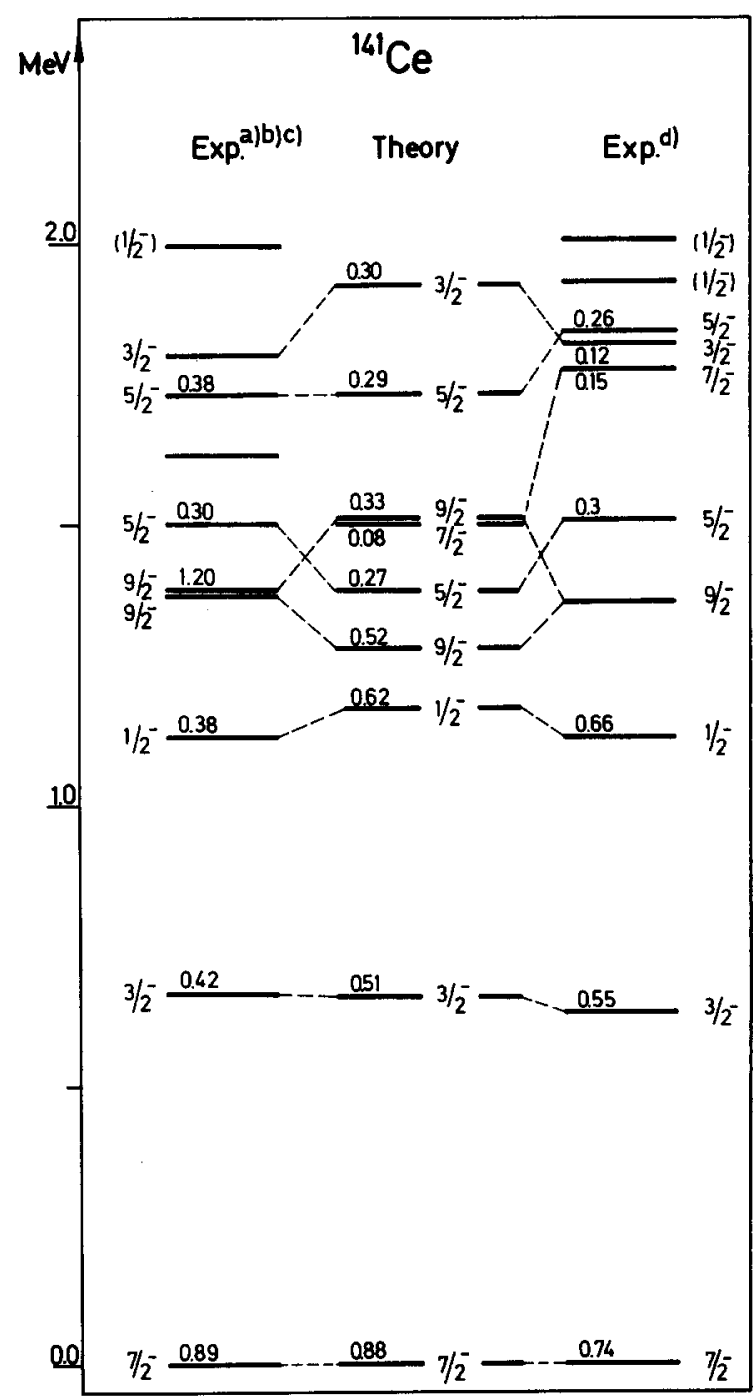

Fig. 1. Experimental and theoretical level schemes of ${ }^{141} \mathrm{Ce}$ up to $2 \mathrm{MeV}$. Corresponding levels are connected. Spectroscopic factors $s(\alpha)$ are also indicated for every level. (a) ref. [7]; (b) ref. [8]; (c) ref. [9]; (d) ref. [3] 
ments. The other $C_{\alpha}(l j, N=1, R=2 ; I)$ are now derived for the first time from proton inelastic scattering experiments to the first $2^{+}$collective excited state $[2,3]$. From these experiments one essentially obtains values for the partialwidth amplitudes $\left(\Gamma_{\alpha}^{l j}\right)^{1 / 2}$ for each channel $\alpha$. These amplitudes carry the spectroscopic information through the following relation

$\left(\Gamma_{\alpha}^{l j}\right)^{1 / 2}=C_{\alpha}(l j, N R ; I)\left[\left(\Gamma_{\alpha}^{\mathrm{s} . \mathrm{p} .}\right)^{l j} /(2 T+1)\right]^{1 / 2}$.

The single-particle widths $\left(\Gamma_{\alpha}^{\mathrm{s} . \mathrm{p} .}\right)^{l j}$ can be obtained from the tables of Thompson [6] at the energy of channel $\alpha$. As we have already calculated the components of the wavefunctions [1] in a unified model where the available singleparticle neutron states $\left(3 p_{1} / 2,3 p_{3} / 2,2 f_{5} / 2\right.$, $2 \mathrm{f} 7 / 2$ and $1 \mathrm{hg} / 2$ ) are coupled to the quadrupole phonon states of a core, it is easy to derive theoretical values for $\left(\Gamma_{\alpha}^{l j}\right)^{1 / 2}$. Experimental values for $\left(\Gamma_{\alpha}^{l j}\right)^{1 / 2}$ are presented in ref. [3] for 6 isobaric analog resonances obtained from the analysis of the reaction ${ }^{140} \mathrm{Ce}\left(p, p^{\prime}\right){ }^{140} \mathrm{Ce}\left(2^{+}\right.$, $1.596 \mathrm{MeV})$. In fig. 1 we give the level schemes as obtained in several experimental works and in the theoretical study. In table 1 we compare the theoretical and experimental partial-widths for the resonances involved. In the analysis of the experimental data the $1 \mathrm{~h}_{9} / 2$ single-particle neutron state is not taken into account, although one may expect an admixture of the following type $[1 \text { h } 9 / 2 \otimes C(12)]^{I M}$ in the states with $I^{\pi}=5 / 2^{-}$ and $7 / 2^{-}$. Proton scattering with $h$ partial waves however are not expected to be observed at the energy used in the experiment through the centrifugal barrier for high $l$ values. As can be seen in table 1 a good overall agreement is obtained between theoretical and experimental data. Practically in all cases the same sign is obtained as derived in experiment. In table 2 we compare the theoretical expansion coefficients with the experimental values [3]. Theoretical values are given for all components of the wavefunctions built on the $0^{+}$groundstate and the first $2^{+}$excited state of ${ }^{140} \mathrm{Ce}$. To be consistent with the experimental data, the $C_{\alpha}(l j, 00 ; j)$ values has been taken positive, and the sign of the other theoretical expansion parameters is adjusted

Experimental and theoretical $\left(\Gamma_{\alpha}^{l j}\right)^{1 / 2}$ values for proton inelastic scattering in the reaction ${ }^{140} \mathrm{Ce}\left(\mathrm{p}, \mathrm{p}^{\prime}\right)$ to the first excited $2^{+}$state. Spins and resonance energies are given.

\begin{tabular}{|c|c|c|c|c|c|c|c|c|c|c|}
\hline \multirow[t]{3}{*}{$I^{\pi}$} & \multirow[t]{3}{*}{$\begin{array}{c}E_{\mathrm{R}}^{\alpha} \\
(\mathrm{MeV})\end{array}$} & \multicolumn{9}{|c|}{$\begin{array}{c}\left.\Gamma_{\alpha}^{l j}\right)^{1 / 2} \\
(\mathrm{keV})^{1 / 2}\end{array}$} \\
\hline & & \multirow[t]{2}{*}{$n l j$} & \multicolumn{2}{|c|}{$3 p_{1 / 2}$} & \multicolumn{2}{|c|}{$3 \mathrm{p}_{3 / 2}$} & \multicolumn{2}{|c|}{$2 f_{5 / 2}$} & \multicolumn{2}{|c|}{$2 \mathrm{f}_{7 / 2}$} \\
\hline & & & exp. & th. & exp. & th. & exp. & th. & exp. & th. \\
\hline $7 / 2^{-}$ & 9.74 & & & & & -0.70 & & -0.09 & & 0.60 \\
\hline $3 / 2^{-}$ & 10.40 & & -0.9 & -0.70 & -0.9 & -0.90 & -0.1 & -0.24 & -2.2 & -1.60 \\
\hline $1 / 2^{-}$ & 10.80 & & & & 2.6 & 4.63 & -1.8 & -0.87 & & \\
\hline $5 / 2^{-}$ & 11.27 & & -2.7 & -0.93 & -0.4 & 0.89 & -1.9 & -0.50 & 2.2 & 2.33 \\
\hline $7 / 2^{-}$ & 11.53 & & & & 1.7 & -0.22 & -0.2 & 0.07 & 4.0 & 3.20 \\
\hline $3 / 2^{-}$ & 11.60 & & 0.5 & -0.90 & -1.0 & -1.45 & -1.0 & -0.21 & 1.1 & 2.65 \\
\hline $5 / 2^{-}$ & 11.62 & & -0.6 & -1.17 & 1.9 & 1.12 & 0.2 & -0.61 & -1.5 & -2.49 \\
\hline
\end{tabular}

Table 2

Spins, excitation energies and expansion coefficients for seven states in ${ }^{141} \mathrm{Ce}$.

\begin{tabular}{|c|c|c|c|c|c|c|c|c|c|c|c|c|c|c|}
\hline \multirow{3}{*}{$\begin{array}{c}E_{\mathrm{X}}^{\alpha} \\
(\mathrm{MeV})\end{array}$} & \multirow{3}{*}{$I^{\pi}$} & \multicolumn{11}{|c|}{$c_{\alpha}(l j, 12 ; I)$} & \multirow{2}{*}{\multicolumn{2}{|c|}{$\mathcal{E}\left|C_{\alpha}(l j, N R ; I)\right|^{2}$}} \\
\hline & & \multicolumn{2}{|c|}{$C_{\alpha}(l j, 00 ; j)$} & \multicolumn{2}{|c|}{$3 \mathrm{p}_{1 / 2}$} & \multicolumn{2}{|c|}{$3 p_{3 / 2}$} & \multicolumn{2}{|c|}{$2 f_{5 / 2}$} & \multicolumn{2}{|c|}{$2 f_{7 / 2}$} & \multirow{2}{*}{$\begin{array}{c}\text { 1hy } / 2 \\
\text { th. }\end{array}$} & & \\
\hline & & th. & exp. & th. & exp. & th. & exp. & th. & exp. & th. & exp. & & th. & exp. \\
\hline 0 & $7 / 2^{-}$ & 0.93 & & & & -0.17 & & -0.04 & & 0.28 & & -0.04 & 0.98 & \\
\hline 0.66 & $3 / 2^{-}$ & 0.72 & 0.83 & -0.15 & -0.19 & -0.20 & -0.20 & -0.08 & -0.05 & -0.60 & -0.84 & & 0.95 & 1.48 \\
\hline 1.14 & $1 / 2^{-}$ & 0.79 & 0.74 & & & 0.44 & 0.53 & -0.30 & -0.58 & & & & 0.91 & 1. 17 \\
\hline 1.53 & $5 / 2^{-}$ & 0.52 & 0.67 & -0.18 & -0.52 & 0.17 & -0.07 & -0.15 & -0.55 & 0.70 & 0.65 & 0.25 & 0.91 & 1.44 \\
\hline 1.79 & $7 / 2^{-}$ & 0.28 & 0.33 & & & -0.04 & 0.31 & -0.02 & 0.04 & 0.87 & 1.07 & 0.00 & 0.84 & 1.27 \\
\hline 1.86 & $3 / 2^{-}$ & 0.55 & 0.40 & -0.17 & 0.10 & -0.26 & -0.18 & -0.06 & -0.26 & 0.70 & 0.29 & & 0.89 & 0.35 \\
\hline 1.88 & $5 / 2^{-}$ & 0.53 & 0.59 & -0.22 & -0.11 & 0.20 & 0.33 & -0.17 & 0.02 & -0.66 & -0.40 & -0.31 & 0.93 & 0.67 \\
\hline
\end{tabular}


relatively to that assumption. In the last column, the sum of the absolute squares of the coefficients is given; this number should by unity if the basis, which has been taken into account, is sufficient. The deviation from unity in the theoretical case is due to the fact that next to the basis states considered in the table, also configurations of two- and three- phonon states coupled with single-particle neutron states have been taken into account. Especially the wavefunction of the $7 / 2^{-}$state at $1.79 \mathrm{MeV}$ has rather large components of the form $\left|2 \mathrm{f}_{7 / 2}, 20 ; 7 / 2\right\rangle, \mid 3 \mathrm{p}_{3 / 2}$, $22 ; 7 / 2\rangle$ and $\left.2 \mathrm{f}_{7 / 2}, 22 ; 7 / 2\right\rangle$ with respective amplitudes $0.18,0.18$ and 0.17 . From the results in table 2 it is straightforward to see that neglecting the 1 hg/ 2 neutron wave in the exit channel in the analysis of the experimental data, is acceptable for all states involved, except for the two $5 / 2^{-}$states, where this wave contributes rather considerably. The experimental $\sum\left|C_{\alpha}(l j, N R ; I)\right|^{2}$ values of the four lowest states, deviate substantially from unity. These deviations are assumed to be due to an underestimation of the single particle-widths. Therefore it would be very interesting if a tabulation of more accurate values for these quantities would become available. However results reported here, indicate that a unified model calculation applied to ${ }^{141} \mathrm{Ce}$, describes rather good the nuclear structure properties, which have been experimentally derived from inelastic scattering of polarized protons from ${ }^{140} \mathrm{Ce}$ to the $2^{+}\left(E_{\mathrm{x}}=\right.$ $1.590 \mathrm{MeV}$ ) state near isobaric analog resonances.

The authors are grateful to Professors J. L. Verhaeghe and C. C. Grosjean for their interest during the course of this work. They are very much indebted to the group of the Physical Institute of the University of Erlangen-Nürnberg for communicating results prior to publication. This investigation was partly supported by the "I. I. K. W. - Brussels, Belgium".

\section{References}

[1] G. Vanden Berghe, K. Heyde and M. Waroquier, Nucl. Phys. A165 (1971) 662.

[2] H. Clement, G. Graw, W. Kretschmer and P. Schulze-Döbold, Phys. Rev. Letters 27 (1971) 526.

[3] H. Clement, thesis, University of ErlangenNürnberg, (1971) (unpublished).

[4] D. Robson, in Isospin in Nuclear Physics, ed. D. H. Wilkinson (North-Holland, Amsterdam 1969) p. 46.

[5] J. P. Wurm, A. Heusler and P. von Brentano, Nucl. Phys. A128 (1969) 433.

[6] W.J. Thompson, Nucl. Data, Sect. A6 (1969) 1.

[7] W. Gelletly, J. A. Moragues, M. A. J. Mariscotti and W. R. Kane, Phys. Rev. C1 (1970) 1052.

[8] C. A. Wiedner, A. Heusler, J. Solf and J. P. Wurm, Nucl. Phys. A103 (1967) 433.

[9] R. H. Fulmer, A. L. McCarthy and B. L. Cohen, Phys. Rev. 128 (1962) 1302. 